\title{
Multirate Simulations With Simultaneous-Solution Using Direct Integration Methods in a Partitioned Network Environment
}

\author{
Fernando A. Moreira, Member, IEEE, José R. Martí, Fellow, IEEE, Luiz C. Zanetta, Jr., Senior Member, IEEE, \\ and Luis R. Linares
}

\begin{abstract}
Multirate simulation of electric networks exhibiting a wide variety of time constants decreases the simulation runtimes by exploiting the property of circuit latency. The fundamental idea is to use different integration steps for different network subsystems, according to the requirements of accuracy of each subsystem. Programs that exploit circuit latency are usually based on relaxation methods that require iterations among the different subsystems of the original networks. These methods lack the numerical robustness of direct implicit integration methods used in circuit simulators and are not adequate for real-time simulation due to their non-uniform solution times. This paper proposes circuit latency exploitation without iterations making use of the concept of interlinked Multi-Area Thevenin Equivalents (MATE). Results are presented showing the efficiency and accuracy of the method.
\end{abstract}

Index Terms-Circuit latency exploitation, multirate simulation, multi-area Thevenin equivalents (MATEs), numerical integration rules.

\section{INTRODUCTION}

$\mathbf{T}$ IME-DOMAIN circuit simulators play an important role in the design and analysis of electric networks. Two of the most successful simulators are SPICE [1] which is normally used for the analysis of electronic and digital circuits and the EMTP [2] which is mainly used in the simulation of power systems. In these simulators the same step size $\Delta t$ is used for the entire network. As a result, the size of $\Delta t$ becomes determined by the requirements of the "fastest" network components. This quite often results in a waste of computational resources since this step size may be unnecessarily small for other parts of the network.

In the 1980's, researchers started to look into the possibility of using, within the same network, two or more different size integration steps. Certain subnetworks are less active or their

Manuscript received February 9, 2005; revised February 7, 2006. The work of F. A. Moreira was supported by the Fundação de Amparo à Pesquisa do Estado de São Paulo (FAPESP), Brazil. This paper was recommended by Associate Editor P. K. Rajan.

F. A. Moreira is with the Electrical Engineering Department, Federal University of Bahia, Salvador, BA, 41830-510, Brazil (e-mail: moreiraf@ufba.br).

J. R. Martí and L. R. Linares are with the Electrical and Computer Engineering Department, University of British Columbia, Vancouver, BC, V6T 1X4, Canada (e-mail: jrms@ece.ubc.ca; 1linares@ece.ubc.ca).

L. C. Zanetta, Jr. is with the Electrical Energy and Automation Engineering Department, University of São Paulo, São Paulo, SP, 05508-900, Brazil (e-mail: lzanetta@pea.usp.br).

Digital Object Identifier 10.1109/TCSI.2006.882821 state variables change very slowly when compared to others. This property is called circuit latency [3].

A number of works have been presented to exploit circuit latency in the time-domain simulation of VLSI circuits and power systems. Most of these works are based on the "waveform relaxation (WR)" technique [4]. In WR each subsystem uses the previous iterate waveforms as "guesses" for the current state of the other subsystems. Iterations are then performed to match the solutions at all interfacing points. Even though successful implementations of WR have been reported [3], [5]-[7], the intrinsic iterative nature of the technique imposes limitations, particularly in the context of real-time simulation and multi-computer simulation environments. The WR advantage in simulation time due to latency exploitation is lost if the solution does not converge quickly. But, more importantly, since the solution time per time step depends on the number of iterations and this number can change from solution point to solution point, the technique is not suited for real time [8]-[10] or multi-computer simulation clusters [11], [12] where the hardware needs to be synchronized at evenly spaced $\Delta t$ time steps with a global clock. In this paper, circuit latency is exploited using a direct simultaneous solution for the branch currents linking the subsystems. Implicit integration methods are used for the circuit equations. The complete network, with different subsystems updated at different $\Delta t$ rates, is solved without iterations. This approach combines the gain in simulation time due to the multirate solution with the robustness of implicit integration methods such as trapezoidal and backward Euler, especially suited for stiff systems.

It should be noted though, that latency exploitation using direct implicit integration methods has been previously proposed both for VLSI circuit and power system simulation. Reference [13] describes a circuit simulator for VLSI simulation using these direct methods. Its main limitation was on how the contribution from the latent part of the network is taken into account when solving only for the fast part. The contribution of the latent part was either completely neglected (as if the circuits were totally decoupled) or computed with a polynomial extrapolation of the last obtained values. Both approaches are severely limited since most latent subcircuits are not completely dormant and polynomial extrapolations are inherently inaccurate for predicting the exponential curves typical in electric networks. In [14], an elaborate implicit integration method is proposed to overcome this limitation. The approach proposed here exploits latency using simple circuit concepts and simple integration techniques such as trapezoidal and backward Euler. 
In the context of power systems, latency exploitation using implicit integration methods has been reported in [15], [16] for transient stability studies and in [17] for electromagnetic transient studies. In [15], the trapezoidal integration rule was used for numerical integration of the fast network components. However, in order to account for the contribution of the latent part of the network, explicit integration methods were used for the slow components, leading to inaccuracies in the simulation. In a subsequent paper [16] the authors claim to have improved their approach by using implicit integration methods for both fast and slow time scales. Although this reference does present important contributions to the subject of multirate simulation in power systems, no explanation is provided on how the influence of the latent part of the network is taken into account using only implicit integration methods. As far as electromagnetic transients simulation is concerned, the possibility of latency exploitation was studied in [17]. Some cases presented in this reference demonstrated the efficiency of latency exploitation for power system transient studies. Resynchronization of the separate solutions for different parts of the network, however, is not made clear in this reference.

Another form of latency exploitation is the utilization of variable time-step methods [18], [19]. The idea is to dynamically adjust the step size during the simulation. If a component is identified as being latent, then its integration step may be increased. Problems arise if the latency condition is not detected properly. For example, if a component is identified prematurely as being latent, then any small error in its value will be propagated to the other components, producing errors in their solutions [3]. A more serious limitation of variable time-step methods occurs if the system presents fast transients sustained for a large portion of the simulation interval. Under these conditions, although only a few of the state variables may be affected by the fast dynamics, the time step must remain small, thus defeating the goal of increased computational efficiency. In addition, variable time-step methods are not suited for real-time simulation due to the needs of synchronized output solutions at evenly spaced $\Delta t$ time steps, as previously mentioned.

Latency exploitation as presented in this paper draws on the conceptual solution framework developed in [20]-[22], known as "multi-area Thevenin equivalents (MATE)." MATE uses the concept of kindred subsystems [20] to split a network into subnetworks of similar solution needs. Each subnetwork is then solved with the technique that is the most efficient for the particular needs of that subnetwork. After each subnetwork has been (separately) solved with its own technique, a Thevenin equivalent of that subnetwork is obtained. The Thevenins of all subnetworks are then brought together and solved for the currents in the link branches connecting the subnetworks. These link currents are then injected back into the individual (isolated) subnetworks to take into account that the subnetwork is not isolated.

Some of the most important earlier work on network partitioning techniques includes the concept of diakoptics developed by Gabriel Kron back in the 1950's [23], [24] and the concept of modified nodal analysis (MNA) by Ho, et al. [25]. The MATE framework partitions the network solution into nodal and branch equations as in MNA, but in MATE the branch equations are means for interfacing subnetworks that have been solved

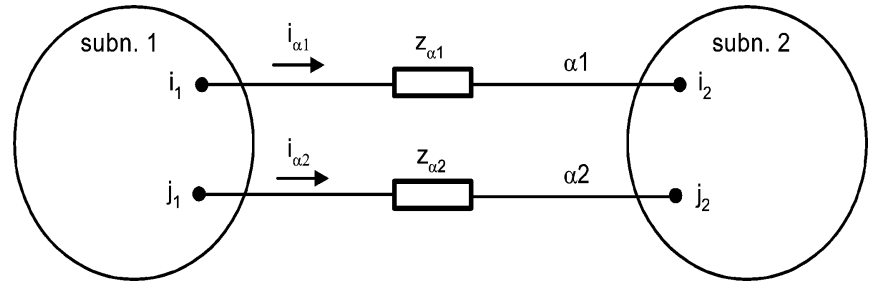

Fig. 1. Sample network for MATE explanation.

with different solution techniques and that are represented by Thevenin equivalents at the moment of performing the simultaneous global solution.

This paper is organized as follows. Section II reviews the derivation of MATE as a reference frame for the presentation of the multirate simulation technique introduced in Section III. Results are presented in Section IV, showing the efficiency and accuracy of the proposed latency method, and finally conclusions are stated in Section V.

\section{MAte PARTitioning SOlUtion}

MATE's conceptual framework and detailed equations are presented in [20]-[22]. Here we review those aspects of the formulation needed to explain the proposed multirate solution. If only $\mathrm{N}$ visible nodes from an internal linear subsystem are needed to connect to an external system, then a Thevenin source vector $\left[E_{\mathrm{th}}\right]_{N \times 1}$ and a Thevenin impedance matrix $\left[Z_{\mathrm{th}}\right]_{N \times N}$ are enough to represent the internal linear subsystem with regards to its interactions with the external system. After the solution of this reduced system of size $N$, voltages and currents in the full internal linear system can be updated by injecting current sources equal to the currents that flow in the links connecting the internal and external system.

Consider the schematic network shown in Fig. 1. This network consists of two subsystems connected by two links identified as $\alpha$. Subsystem 1 has a total of " $m$ " nodes, whereas subsystem 2 has a total of " $n$ " nodes.

The following notation is used.
$\left[A_{1}\right],\left[A_{2}\right]$ Admittance matrices of subnetworks 1 and 2, respectively.
$i_{\alpha 1}, i_{\alpha 2} \quad$ Currents in link branches $\alpha 1$ and $\alpha 2$, respectively.
$i, j \quad$ Nodes in subnetworks 1 and 2 where links $\alpha$ are connected.
$\left[v_{1}\right],\left[v_{2}\right] \quad$ Node voltages of subnetworks 1 and 2, respectively.
$\left[h_{1}\right],\left[h_{2}\right]$ Current nodal injections in subnetworks 1 and 2, respectively.

For the purpose of evaluating the link currents $i_{\alpha 1}$ and $i_{\alpha 2}$, subsystems 1 and 2 may be substituted by Thevenin equivalents: source vectors $\left[E_{\mathrm{th}_{-} 1}\right]_{2 \times 1}$ and $\left[E_{\mathrm{th} \_}\right]_{2 \times 1}$ and impedance matrices $\left[Z_{\mathrm{th}_{-} 1}\right]_{2 \times 2}$ and $\left[Z_{\mathrm{th}_{-} 2}\right]_{2 \times 2}$. The dimensions of the vectors and matrices are in accordance with the number of interconnecting links. 


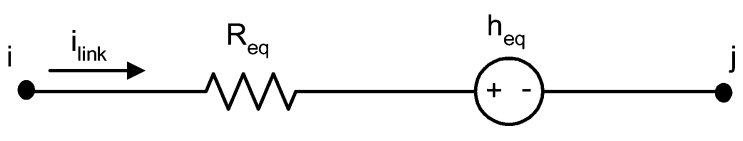

Fig. 2. General equivalent circuit for link representation.

TABLE I

REPRESENTATION OF $R, L$, AND $C$ ELEMENTS

\begin{tabular}{|c|c|c|c|c|}
\hline \multirow{2}{*}{ Element } & \multicolumn{4}{|c|}{ Integration rule } \\
\cline { 2 - 5 } & \multicolumn{3}{|c|}{ Trapezoidal } & \multicolumn{2}{|c|}{ Backward Euler } \\
\cline { 2 - 5 } & $R_{e q}$ & $h_{e q}(t)$ & $R_{e q}$ & $h_{e q}(t)$ \\
\hline$R$ & $R$ & 0 & $R$ & 0 \\
\hline$L$ & $\frac{2 L}{\Delta t}$ & $-v_{L}(t-\Delta t)-\frac{2 L}{\Delta t} i_{L}(t-\Delta t)$ & $\frac{L}{\Delta t}$ & $-\frac{L}{\Delta t} i_{L}(t-\Delta t)$ \\
\hline$C$ & $\frac{\Delta t}{2 C}$ & $v_{C}(t-\Delta t)+\frac{\Delta t}{2 C} i_{C}(t-\Delta t)$ & $\frac{\Delta t}{C}$ & $v_{C}(t-\Delta t)$ \\
\hline
\end{tabular}

\section{A. MATE's Nodal Equations}

If none of the links indicated in Fig. 1 existed, we would have two independent subnetworks, 1 and 2 . The corresponding nodal equations would be as follows

$$
\left[\begin{array}{cc}
A_{1} & 0 \\
0 & A_{2}
\end{array}\right]\left[\begin{array}{l}
v_{1} \\
v_{2}
\end{array}\right]=\left[\begin{array}{l}
h_{1} \\
h_{2}
\end{array}\right] .
$$

Branch equations should now be added in order to represent the links in the original interconnected network.

\section{B. MATE's Branch Equations}

For discrete-time solutions, the branch equations have the general form

$$
v_{i}(t)-v_{j}(t)=R_{\mathrm{eq}} \cdot i_{\mathrm{link}}(t)+h_{\mathrm{eq}}(t)
$$

corresponding to the equivalent circuit represented in Fig. 2, where

$R_{\text {eq }} \quad$ discretized equivalent resistance (dependent on the integration rule);

$h_{\text {eq }}$ equivalent history voltage source (dependent on the integration rule).

The value of $R_{\mathrm{eq}}$ and $h_{\mathrm{eq}}$ depend on the type of element and on the integration rule used (e.g., trapezoidal, backward Euler). This representation is very well known in circuit simulation programs like the EMTP and SPICE. Table I summarizes the representation of basic $R, L$, and $C$ elements.

Clearly, any series combination of $R, L$, and $C$ elements can also be implemented as a link just making sure that the correct equivalent resistance and history voltage source are calculated.

Two special conditions may also occur for the link.

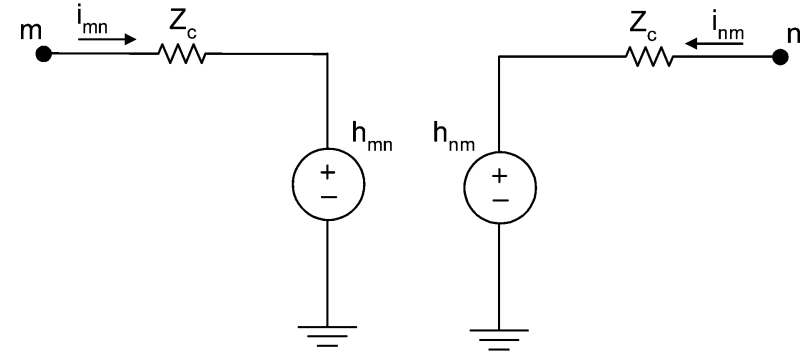

Fig. 3. Model of a transmission line.

1) Link is a Short Circuit: This is a special case where both $R_{\text {eq }}$ and $h_{\text {eq }}$ are zero and the branch equation is simply

$$
v_{i}(t)-v_{j}(t)=0
$$

2) Link is a Transmission Line Modeled With the Bergeron's Method (Method of Characteristics): Fig. 3 shows the Bergeron's model of a transmission line between two general nodes [26]. The line has a characteristic impedance $Z_{c}$ and a travel time $\tau$. The voltage sources $h_{m n}(t)$ and $h_{n m}(t)$ are given by

$$
\begin{aligned}
& h_{m n}(t)=v_{n}(t-\tau)+Z_{c} i_{n m}(t-\tau) \\
& h_{n m}(t)=v_{m}(t-\tau)+Z_{c} i_{m n}(t-\tau) .
\end{aligned}
$$

The transmission-line model effectively decouples the subnetworks connected to the sending and receiving ends of the line. Therefore, no branch equation needs to be written when the network decoupling is performed through a transmission line.

\section{MATE's Combined Nodal and Branch Equations}

The node equations of subnetworks 1 and 2 can be combined with the branch equations in a "common container" as follows:

$$
\left[\begin{array}{ccc}
A_{1} & 0 & p \\
0 & A_{2} & q \\
p^{t} & q^{t} & -z_{\alpha}
\end{array}\right]\left[\begin{array}{c}
v_{1} \\
v_{2} \\
i_{\alpha}
\end{array}\right]=\left[\begin{array}{c}
h_{1} \\
h_{2} \\
h_{\mathrm{eq} \alpha}
\end{array}\right]
$$

where

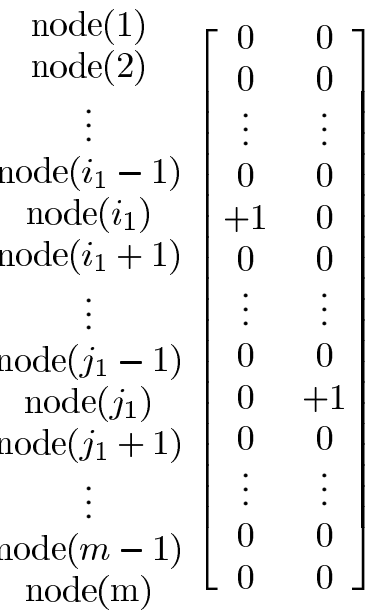




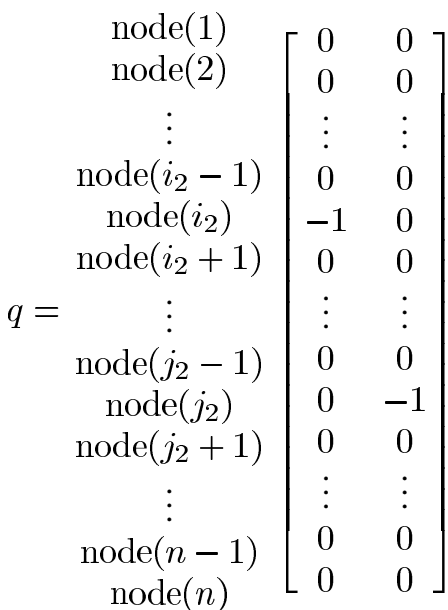

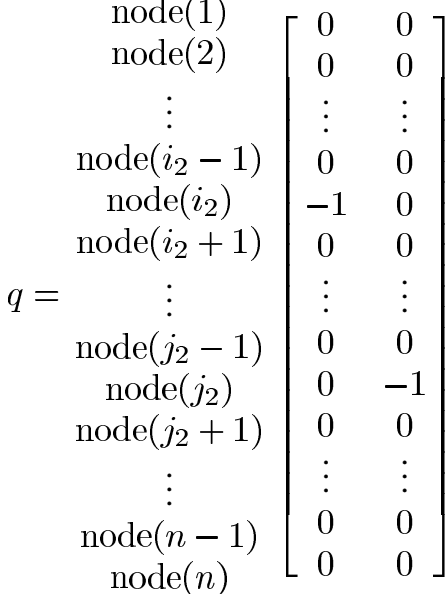

$$
\begin{aligned}
& z_{\alpha}=\left[\begin{array}{cc}
z_{\alpha 1} & 0 \\
0 & z_{\alpha 2}
\end{array}\right] \text {. }
\end{aligned}
$$

Multiplying the first two rows of (5) respectively by $A_{1}^{-1}$ and $A_{2}^{-1}$, this equation can be rewritten as

$$
\left[\begin{array}{ccc}
1 & 0 & a \\
0 & 1 & b \\
0 & 0 & Z_{\alpha}
\end{array}\right]\left[\begin{array}{l}
v_{1} \\
v_{2} \\
i_{\alpha}
\end{array}\right]=\left[\begin{array}{l}
e_{1} \\
e_{2} \\
e_{\alpha}
\end{array}\right]
$$

where

$$
\begin{aligned}
a & =A_{1}^{-1} p \\
b & =A_{2}^{-1} q \\
e_{1} & =A_{1}^{-1} h_{1} \\
e_{2} & =A_{2}^{-1} h_{2} \\
Z_{\alpha} & =p^{t} a+q^{t} b+z_{\alpha} \\
e_{\alpha} & =p^{t} e_{1}+q^{t} e_{2}-h_{\mathrm{eq} \alpha} .
\end{aligned}
$$

The link currents are obtained from the last row in (8)

$$
\left[Z_{\alpha}\right]_{2 \times 2}\left[i_{\alpha}\right]_{2 \times 1}=\left[e_{\alpha}\right]_{2 \times 1}
$$

The components of $\left[e_{\alpha}\right],\left[p^{t} e_{1}\right]$ and $\left[q^{t} e_{2}\right]$ are respectively the Thevenin source vectors $\left[E_{\mathrm{th} \_}\right]$and $\left[E_{\mathrm{th}} 2\right]$. Similarly, the components of $\left[Z_{\alpha}\right],\left[p^{t} a\right]$ and $\left[q^{t} b\right]$ are respectively the Thevenin impedance matrices $\left[Z_{\mathrm{th}_{-} 1}\right]$ and $\left[Z_{\mathrm{th} \_2}\right]$.

For the complete MATE solution, the following procedure is adopted.

1) Obtain the steady-state solution of the complete network (at $t=0$ ).

2) Build the admittance matrices for subnetworks 1 and 2 as if they were completely independent.

3) Advance the solution time by $t=t+\Delta t$.

4) Update the history source terms for subnetworks 1 and 2.

5) Solve for the internal node voltages of subnetworks 1 and 2 still considering both completely decoupled.

6) Solve for the link currents $i_{\alpha 1}$ and $i_{\alpha 2}$ using the node voltages calculated in step 5 .

7) Update the internal node voltages of subnetworks 1 and 2 by injecting the link currents $i_{\alpha 1}$ and $i_{\alpha 2}$ into the corresponding nodes.

8) Go back to step 3 until $t=t_{\max }$.

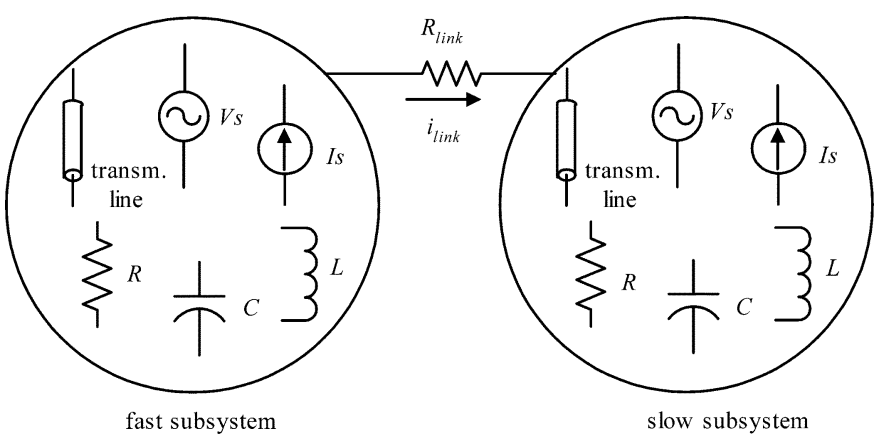

Fig. 4. Interconnection of a fast and slow subsystem.

\section{Direct Method CirCuit LATENCY EXPloitation UNDER MATE'S FRAMEWORK}

MATE's concept of linked Thevenins provides the framework for latency exploitation with simultaneous solution discussed in this paper and introduced previously in [27], [28]. If the part of the network that needs to be simulated in detail (with a small time step) is isolated into a subsystem, whereas the rest of the network forms one or more subsystems, branch tearing can be performed at the links connecting the distinct subsystems. The multinode Thevenin equivalent reduction process can then be applied to the subsystems. Since our main concern here is to show how the interconnection of the fast and slow subnetworks is dealt with, only two subsystems are considered throughout this section: one representing the fast subnetwork and the other representing the slow one. However, the application to any number of subnetworks can be performed in a straight forward way by following the derivation presented in Section II. Fig. 4 depicts a schematic diagram showing the interconnection of a fast and slow subsystem through a pure resistance. Each subsystem contains any combination of lumped and distributed elements as well as voltage and current sources.

An important observation should be made before we proceed. It is often not true that individual subsystem components can be characterized in an absolute way as being either "slow" or "fast," since normally all dynamic modes are present in all state variables. Even if slow and fast modes are clearly identified in a general lumped $R L C$ network it may be difficult to identify state variables as depending predominantly on slow modes. If a state variable is incorrectly supposed to be slow but actually has a strong dependency on fast modes, then latency exploitation may result in erroneous results. It is not within the scope of this paper to present a generalization of the latency method for general lumped networks. Some ideas, however, have been presented in [29] where the calculation of the "participation matrix" is proposed in order to verify if the network partitioning is adequate for latency exploitation. The participation matrix only depends on the right and left eigenvectors associated with each mode of the system and each element of this matrix is a measure of the influence of each mode in each state variable. It is then possible to verify if the network partitioning employed would provide accurate results or not.

The multirate method as presented in this paper is therefore intended for networks where there are some truly valid, perhaps physically based, reasons for considering a subsystem to 


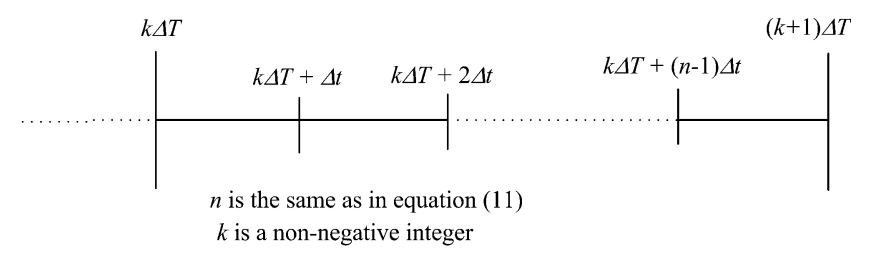

Fig. 5. Timeline of the simulation at a general time interval when latency is exploited.

be "slow." Fortunately, in many practical circuits varying from VLSI designs to power systems applications, this assumption is generally true. In the power systems field, high-voltage direct current (HVDC) converters and pulsewidth modulated (PWM) inverters are examples where it is known that the system can be split into fast and slow subsystems. Transmission lines also usually allow the linking of a fast and slow subsystem as long as the time step employed in the slow subsystem is less than the traveling time of the line. In the next section, simulation results will be shown for these case examples.

For the sake of simplicity, the large integration step is defined as an integer multiple of the small one

$$
\Delta T=n \Delta t
$$

where $n$ is a positive integer, larger than unity and $\Delta T$ and $\Delta t$ are, respectively, the large and small time steps.

Fig. 5 shows the timeline of the simulation at a general time interval.

At instants multiple of the large time step $\Delta T$, both the slowvarying and the fast-varying subsystems are solved. These are the instants when the solutions are resynchronized. At the small time steps $\Delta t$ within the large step $\Delta T$ only the fast-varying subsystem is solved. Care should be taken on determining how to represent the slow subsystem when only the fast one is solved. Since slow-varying subnetworks are not in general completely latent it would be incorrect to entirely neglect their contribution for the solution of the fast-varying subnetworks. Also, when the solution is resynchronized, i.e., when the entire network is solved, the variation of the fast subnetwork solution through a complete $\Delta T$ interval should be taken into account for the solution of the slow subnetwork. This way, significant sampling errors may be prevented or at least reduced [30]. The solutions proposed to overcome these difficulties will now be presented in detail.

\section{A. Thevenin Equivalent Interpolation for the Fast-Varying Subsystem Solution}

As previously discussed, a critical aspect of exploiting latency is the evaluation of the contribution of the latent subsystems to the solution of the fast-varying subsystems. There have been approaches where the effects of latent subcircuits have been modeled with polynomial extrapolation [13] or by developing elaborate implicit integration methods [14]. However, numerical extrapolations are inherently inaccurate for predicting solutions in fast-varying electrical networks, and our focus is to exploit latency using simple implicit integration methods like trapezoidal and backward Euler. Through the framework provided by the MATE technique, it is possible to take into account

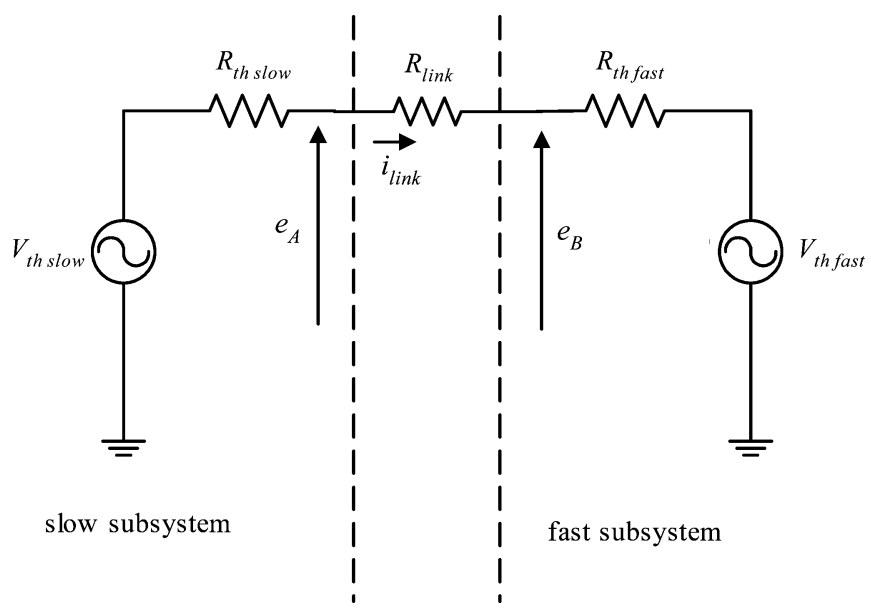

Fig. 6. Thevenin equivalent representation of slow and fast subnetworks at linking nodes.

the contribution of the latent subcircuit using only numerical interpolation for linear systems. Alternatives for nonlinear systems are discussed in the conclusions and in the Appendix.

From Fig. 4, it is readily observed that the link current can be calculated if both subsystems are first transformed into their respective Thevenin equivalents, as discussed in Section II. This representation is shown in Fig. 6. The Thevenin equivalent voltage source represents the combined effects of the independent voltage and current sources together with the history sources existent in the original network.

After a particular solution instant, when the complete network has been solved, there will be a number of solution steps (depending on the ratio $\Delta T / \Delta t$ ) when only the fast subsystem needs to be solved. Thus, the representation of the slow subsystem as a Thevenin equivalent seems to be very adequate. If the Thevenin equivalent were to be maintained constant throughout the simulation interval $\Delta T$, the latent subnetwork would be represented as completely dormant, resulting in a possibly inaccurate solution. If, however, a varying Thevenin equivalent is employed for the latent subnetwork at each small time step $\Delta t$, accuracy can be significantly increased. The network representation of Fig. 6 allows for latency exploitation using noniterative solution methods and numerical interpolation techniques instead of extrapolations. The link current at any particular time instant can be calculated as

$$
i_{\text {link }}=\frac{e_{A}-e_{B}}{R_{\text {link }}}
$$

and once the link current is obtained, the internal node voltages can be updated by following the MATE technique.

A varying Thevenin equivalent can be easily implemented as follows. The Thevenin equivalent voltage source of the slow subnetwork $\left(V_{\text {thslow }}\right)$ is a function of the independent voltage and current sources, and the history sources within this subnetwork. It can be expressed mathematically as

$$
V_{\text {thslow }}(t)=f\left(v_{s}(t), i_{s}(t), e_{\mathrm{hs}}(t)\right)
$$

where $\boldsymbol{v}_{s}(\cdot)$ is the vector of all independent voltage sources, $\boldsymbol{i}_{s}(\cdot)$ is the vector of all independent current sources, and $\boldsymbol{e}_{\mathrm{hs}}(\cdot)$ is the vector of all history sources within the subnetwork. The 


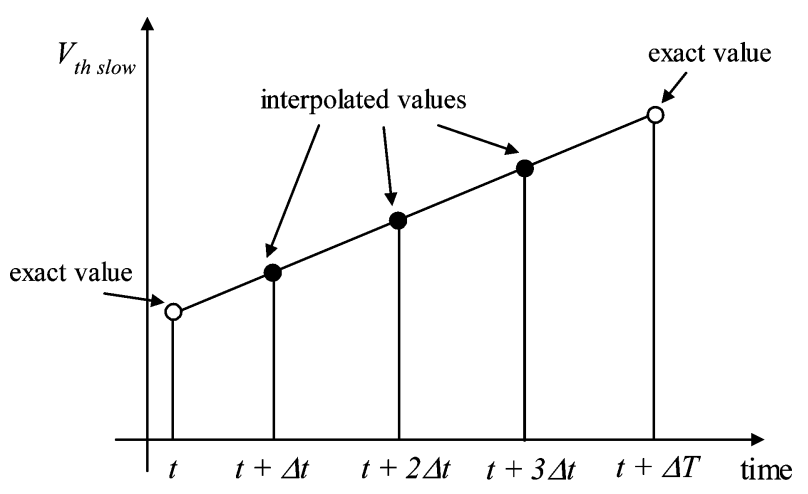

Fig. 7. Thevenin equivalent voltage source linear interpolation for $\Delta T=4 \Delta t$.

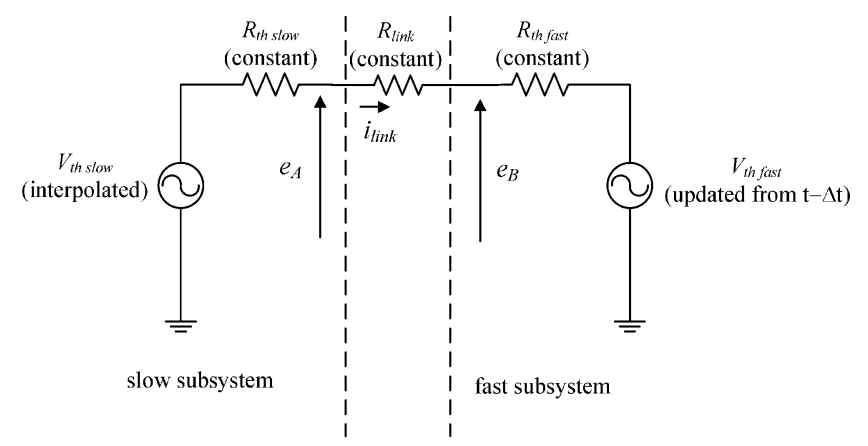

Fig. 8. Indication of how each network component is calculated when only the fast subnetwork is solved in detail.

history sources only depend on the node voltages and branch currents determined at the previous large time step $\Delta T$ as in

$$
e_{\mathrm{hs}}(t)=g(v(t-\Delta T), i(t-\Delta T))
$$

where $\boldsymbol{v}(\cdot)$ is the vector of known node voltages and $\boldsymbol{i}(\cdot)$ is the vector of known branch currents.

As soon as the global solution is obtained at, for example, $t=$ $n \Delta T$, the history sources of the slow varying elements at $t=$ $(n+1) \Delta T$ may be evaluated by applying (14). Also, the values of the independent voltage and current sources are a known function of time. Therefore, after substituting (14) in (13), it is possible to evaluate the Thevenin equivalent voltage source of the slow subnetwork at $t=(n+1) \Delta T$ without having to run through the small integration steps $\Delta t$.

After determining the value of the Thevenin equivalent voltage source of the slow subnetwork at $t=(n+1) \Delta T$, it is possible to calculate the values to be used for each small integration steps $\Delta t$ within the large time period $\Delta T$ through a simple interpolation process. With the assumption that the latent subnetwork is varying slowly throughout the interval $\Delta T$ (otherwise the integration step $\Delta T$ itself would have to be decreased), a linear interpolation of the Thevenin equivalent voltage source is adopted. This procedure is consistent with the trapezoidal discretization solution. Fig. 7 shows how the interpolation procedure works, assuming, for example, $\Delta T=4 \Delta t$.

In Fig. 8 the network representation of Fig. 6 is repeated but now with the indication of how each network component is represented for the calculation of the link current at time instants when only the fast subnetwork is solved in detail.

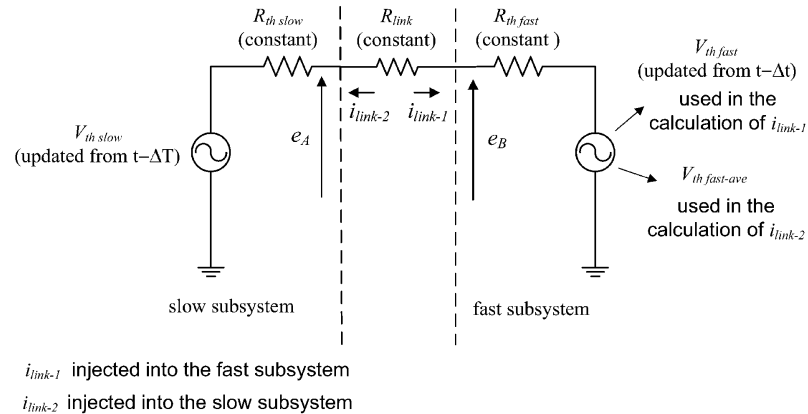

Fig. 9. Network representation for complete network solution.

\section{B. Double Link Current Injection at Resynchronized Solution Points}

Whenever it is time to solve for the entire network (at every instant multiple of the large time step $\Delta T$ ) the history sources for both the slow-varying and the fast-varying subnetworks should be updated. For the slow-varying subnetwork, the last solution was calculated at the instant $t-\Delta T$ so the history sources are naturally updated with the values at this time instant. The fast-varying subnetwork however, has been solved $n$ times, according to (11) during a complete $\Delta T$ cycle. The last calculated solution at $t-\Delta t$ is therefore not representative of the fast subsystem's variation from the viewpoint of the slow subsystem. An average of the solution of the fast subsystem variables obtained through the complete $\Delta T$ cycle would be more accurate. On the other hand, the evaluation of an average for all fast variables would require many calculations, therefore, defeating the very purpose of latency exploitation. A compromise solution may be reached by averaging only the Thevenin equivalent voltage source of the fast subnetwork $V_{\text {thfast }}$ (which is a fast variable anyway) and using it to update the node voltages of the slow subnetwork.

The way to implement this solution is to perform a double system update at the resynchronization instants. The history sources of the fast components are updated from the solution obtained at $t-\Delta t$, i.e., from the last calculated solution for this part of the network. Different link currents are then evaluated according to the part of the network that is going to be solved. For the fast subnetwork solution the link current is simply calculated according to (12). However, for the slow subnetwork, first the average of $V_{\text {thfast }}$ is obtained through a complete $\Delta T$ cycle, so that the variation of the fast-varying elements during the $\Delta T$ cycle is taken into account. This approach prevents the quasi-randomness of taking into account only the single value of the last calculated solution of the fast subnetwork. This average of $V_{\text {thfast }}\left(V_{\text {thfast-ave }}\right)$ is calculated as follows:

$$
V_{\text {thfast-ave }}=\frac{\sum_{k=1}^{n} V_{\text {thfast }}(t-\Delta T+k \Delta t)}{n} .
$$

The link current that is going to be injected into the slow subsystem is now calculated with $V_{\text {thfast }}$ substituted by $V_{\text {thfast-ave }}$. Fig. 9 illustrates the situation.

Although only two different time scales are addressed in this formulation, the extension of the latency technique proposed in this paper to multiple time scales is quite straight forward as 


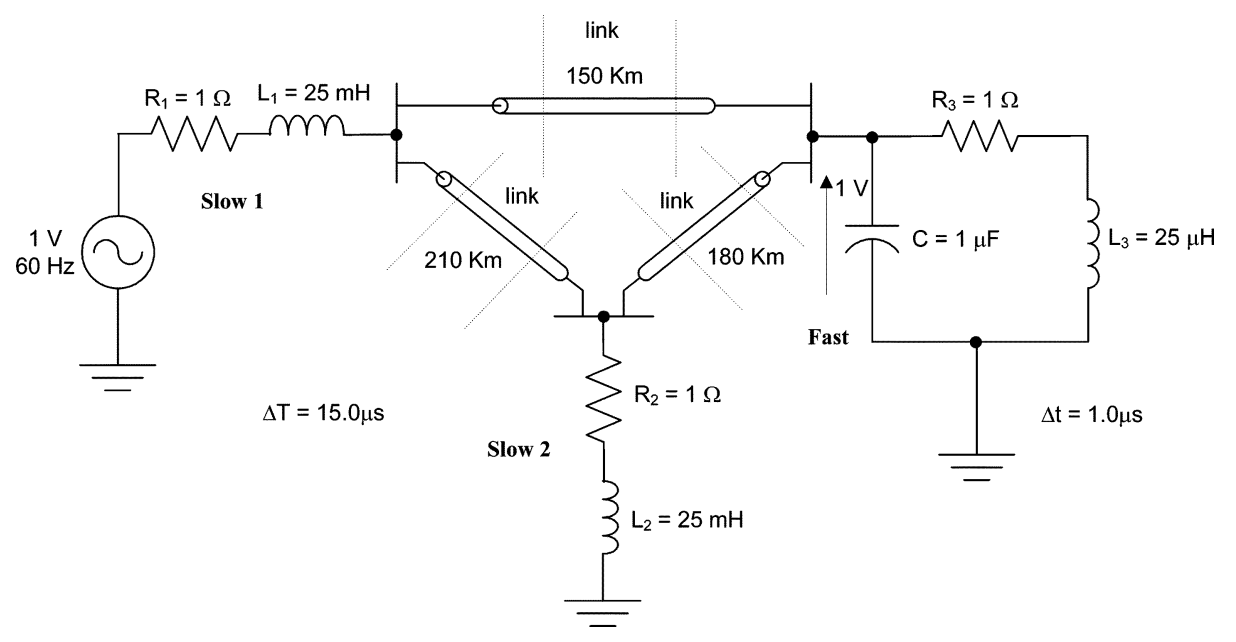

Fig. 10. Electric network with lossy lines for latency exploitation.

long as the time steps form a sequence in which the smallest integration step is a submultiple of the second smallest one, which is in turn a submultiple of the third smallest one, and so on. Only at time instants multiples of the large integration step would the complete network be solved together. At all other instants, those subnetworks with an integration step that is a submultiple of the actual time instant would be solved in the way shown in Fig. 8 for the fast subsystem, in this particular situation of double-rate simulation. Other subnetworks would be represented by their corresponding interpolated $V_{\text {thslow }}$, as also shown in Fig. 8 for the double rate simulation case. Future work is intended in order to allow the use of time steps that do not follow this pattern.

\section{Simulation Results}

\section{A. Latency Exploitation on Circuits Containing Lossy Transmission Lines}

The circuit shown in Fig. 10 was simulated. This circuit contains two slow areas and one fast area interconnected by lossy transmission lines. The system is energized at $t=0$ by applying a sinusoidal voltage with frequency $60 \mathrm{~Hz}$ and amplitude $1.0 \mathrm{~V}$. The fast transients are triggered by applying an initial charge of $1.0 \mathrm{~V}$ to the capacitor of the fast load.

The single phase transmission lines are the links of the network and the fast and slow areas are simulated with different integration step sizes. The lengths of each transmission line are indicated in Fig. 10. They have been modeled as lossy by lumping its total distributed resistance half at each line end. The line model itself is as indicated in Fig. 3. The time step employed for the slow parts is $15.0 \mu \mathrm{s}$, while the time step adopted for the fast part is $1.0 \mu \mathrm{s}$ in order to represent the fast dynamics accurately. The trapezoidal integration rule has been adopted for this circuit simulation. Fig. 11 shows the voltage obtained across the fast capacitor for both the single-time-step and the two-time-steps solution.

The results obtained with the double- time-step method are indistinguishable from those obtained with the single step one. Since the transmission lines are lossy, the fast transient is

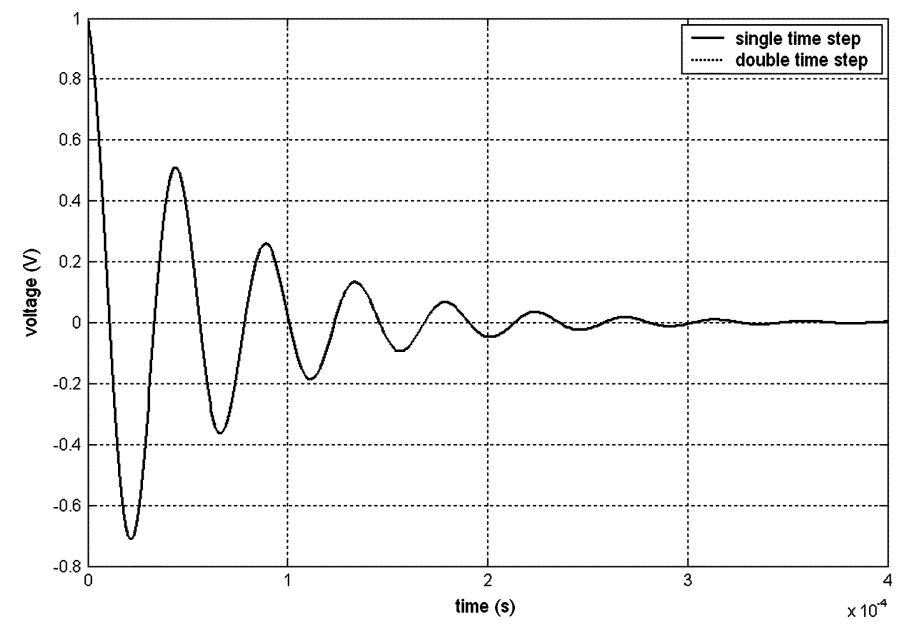

Fig. 11. Voltage across the fast capacitor for the single-time-step $(\Delta t=1.0 \mu \mathrm{s})$ and for the two-time-steps solutions $(\Delta T=15.0 \mu \mathrm{s}$ and $\Delta t=1.0 \mu \mathrm{s})$.

strongly attenuated as it travels down the lines. For this reason, the voltages at the slow part of the network will not present any significant high-frequency components. Fig. 12 shows the voltage across the inductance located in the slow part 1 again for the two distinct simulation cases. The results agree quite well despite a faster decay of the voltage for the two-time-steps case at the initial transient.

It should be emphasized that if the transmission lines were lossless, the fast transient would have propagated with no attenuation to the slow areas. In this case, the latency methodology would not accurately reproduce the fast transients superimposed on the slow system; it would superimpose the average of their effect instead. In many cases, the average of the fast transients is all that is needed in the slow system. In order to show this point, Fig. 13 depicts once again the voltage across the inductance located in the slow part 1 now considering the lines to be lossless.

Although the fast transient cannot be reproduced with a high degree of accuracy, especially the steep peak at $t=0.5 \mathrm{~ms}$, the method proposed is capable of following the behavior of 


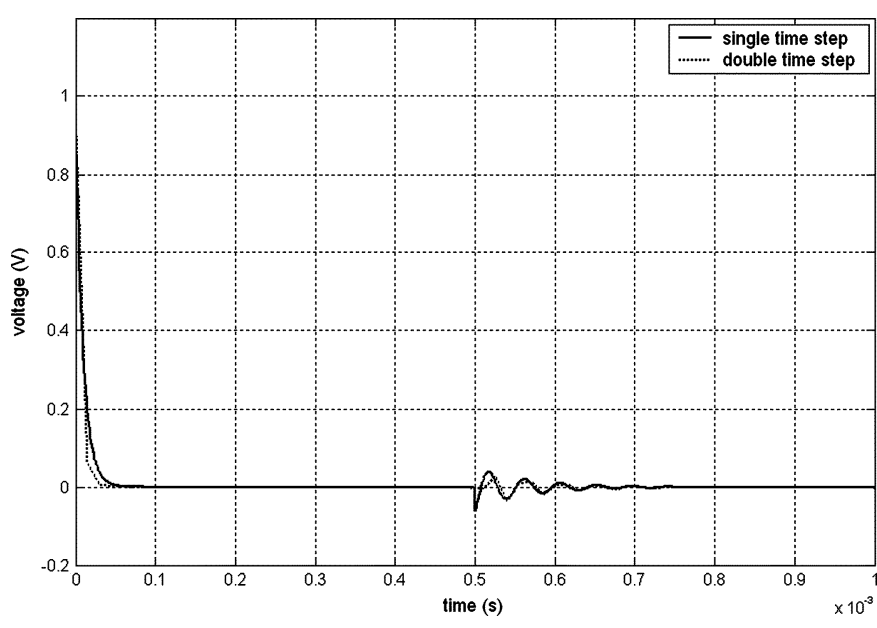

Fig. 12. Voltage across the inductor in slow area 1 for the single-time-step $(\Delta t=1.0 \mu \mathrm{s})$ and for the two-time-steps solutions $(\Delta T=15.0 \mu \mathrm{s}$ and $\Delta t=1.0 \mu \mathrm{s})$.

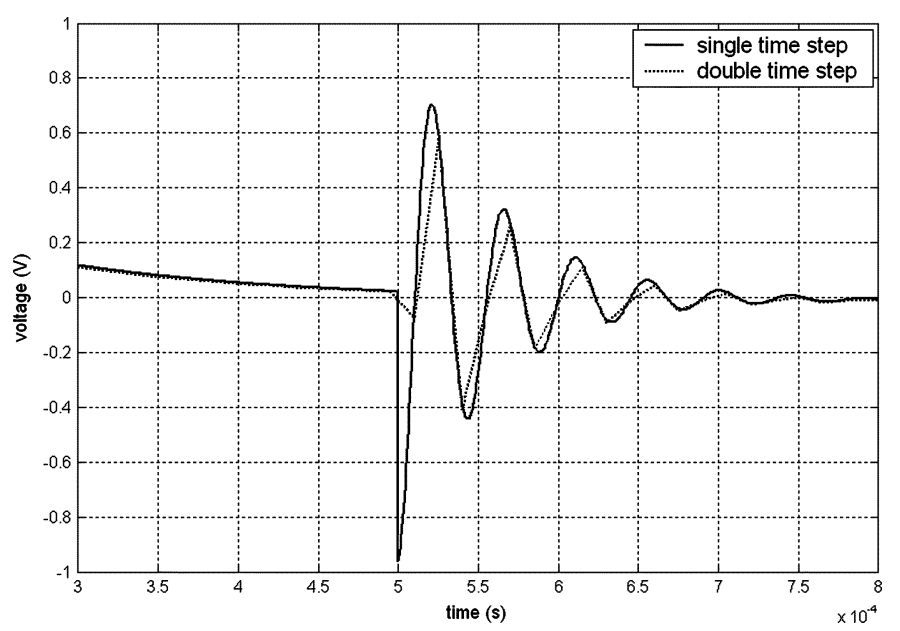

Fig. 13. Voltage across the inductor in slow area 1 for the single-time-step $(\Delta t=1.0 \mu \mathrm{s})$ and for the two-time-steps solutions $(\Delta T=15.0 \mu \mathrm{s}$ and $\Delta t=1.0 \mu \mathrm{s})$. Treating the lines as lossless.

the signal in a much better way than if the simulation were performed with a large time step of $15.0 \mu \mathrm{s}$. Fig. 14 shows the results obtained in this situation.

The double- time-step method is capable of following relatively well the time constant associated with the attenuation of the fast transient, something that is not possible to attain when simulating the network with a single and large integration step.

An assessment of the computational complexity is needed in order to verify the efficiency of latency exploitation. This particular network contains four elements for which history sources must be evaluated (three inductances and one capacitance). Six history sources must also be evaluated for the transmission lines (two for each line). When a simulation with a single time step is performed, these ten history sources must be evaluated at every step. If the simulation is performed with the two-timesteps method, then only four history sources would need to be updated at every small time step $\Delta t$ (the capacitance and the inductance located in the fast part of the network plus the two history sources for each of the transmission lines terminating in this fast part). In terms of node voltages, the whole network

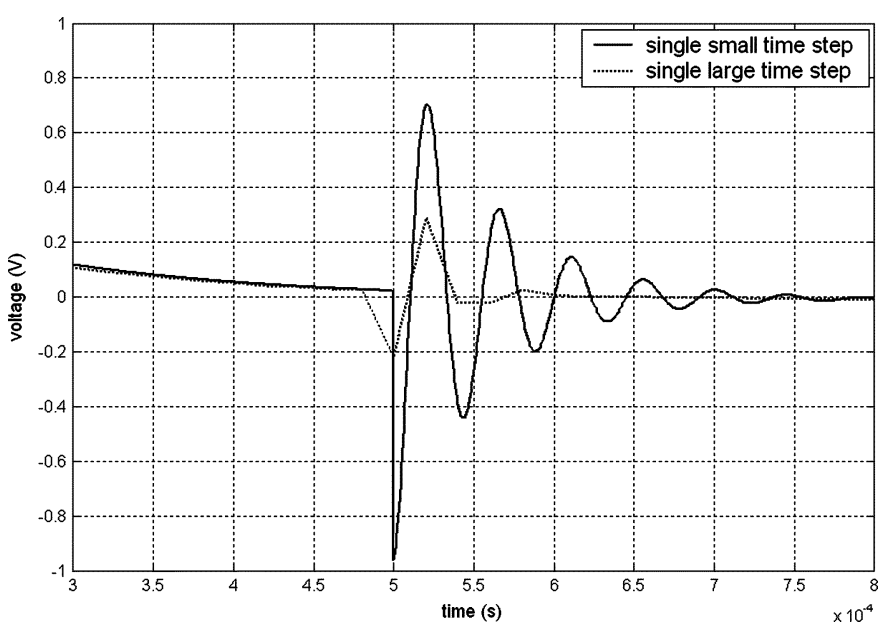

Fig. 14. Voltage across the inductor in slow area 1 for the single small time step $(\Delta t=1.0 \mu \mathrm{s})$ and for the single large time-step solutions $(\Delta T=15.0 \mu \mathrm{s})$. Treating the lines as lossless.

TABLE II

NuMBer of FLOPS FOR Single- AND DOUble-Time-STEP Methods. SIMULATION RUN UP TO 1.0 MS

\begin{tabular}{|c|c|}
\hline Method & FLOPS \\
\hline Single time step $(\Delta t=1.0 \mu \mathrm{s})$ & 170,116 \\
\hline Double time step $(\Delta t=1.0 \mu \mathrm{s}$ and $\Delta T=15.0 \mu \mathrm{s})$ & 99,547 \\
\hline
\end{tabular}

contains a total of twelve nodes with unknown voltage values (it must be remembered that in comparison with the figure some more nodes appear due to the lumping of the transmission line resistances at each end of the lines). Among these twelve nodes, eight are located within the slow parts of the network and are evaluated only at every large step $\Delta T$. The other four nodes are located within the fast part of the network and need to be calculated at every small time step $\Delta t$. It should be remembered that the Thevenin equivalent voltage sources for each of the slow parts of the network are evaluated at every small time step, as explained in Section III-A. Table II shows the number of floatingpoint operations performed for each situation in order to execute the simulation up to $1.0 \mathrm{~ms}$. The codes have been written in MATLAB version 6, both for the double- and single-time-step methods for fairness of comparison.

The single-time-step alternative requires approximately $70 \%$ more FLOPS than the double-time-step case even for this small network. The latency method will show significant more gains compared to the traditional single-step simulation in the case of larger networks containing, for example, several transmission lines separating a fast subnetwork from the rest of the system.

\section{B. Latency Exploitation on a Rectifier Bridge}

Power electronics-based networks are usually well suited for multirate simulations since the switching devices operate at frequencies much higher than the remaining part of the network. To correctly represent the dynamics of the switching valves, a time step of $50 \mu \mathrm{s}$ is normally adopted in order to correspond to approximately one electrical degree at a frequency of $60 \mathrm{~Hz}$. However, adopting such a small time step for the complete network may result in a significant waste of computational resources, 


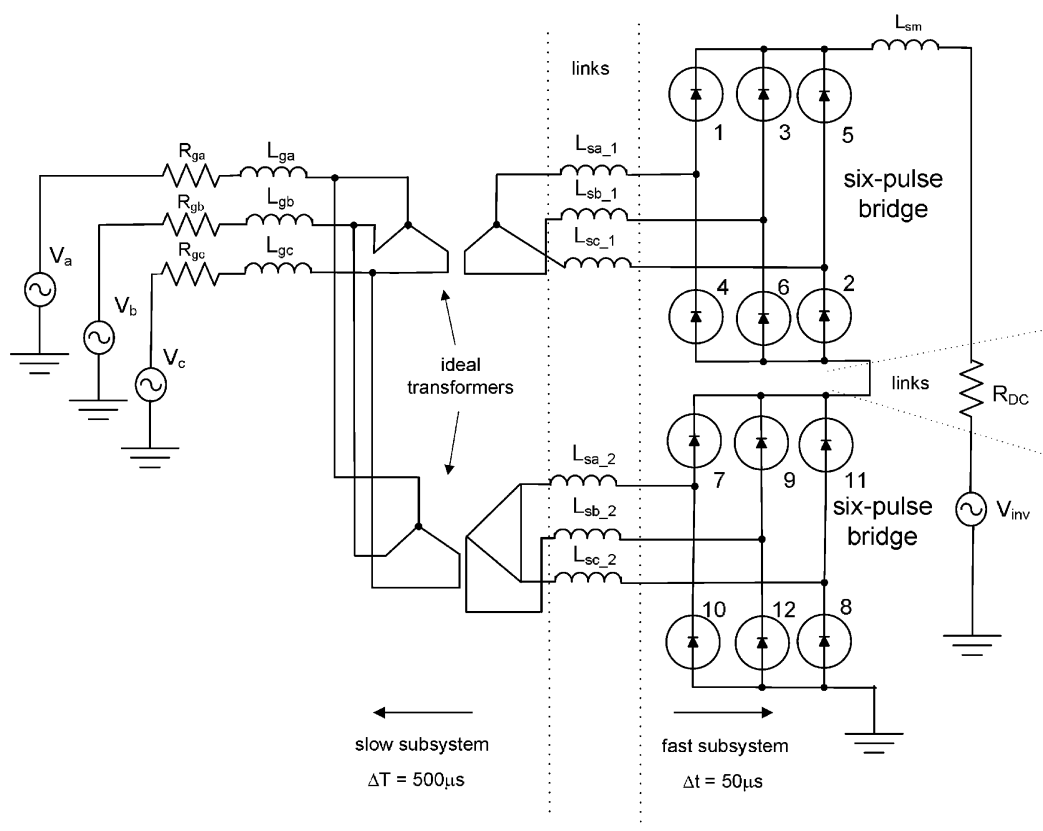

Fig. 15. Three-phase twelve-pulse rectifier bridge.

since the rest of the network could usually be simulated with much larger step sizes, in the order of hundreds of microseconds. Reference [31] describes an approach where the time step size is reduced for the converter part of the network whenever a commutation is detected. The authors however, have used a state variable formulation to integrate the differential equations of the converter. Although this approach provides the flexibility of readily changing the time step during a run, it has the inherent drawback associated with the difficulty of writing code for the generation of state equations given the network connectivity information. For proper processing, graph theory must be used, which requires a special sequence in which the elements are taken to form the normal tree [32].

If the modified nodal formulation is used instead, as it is currently done in almost all circuit simulators, then the approach described in [31] would have the limitation of requiring the recalculation of the whole conductance matrix whenever a switching is detected.

In this paper, latency is exploited on a three-phase twelvepulse diode rectifier bridge, commonly employed for HVDC transmission (in fact thyristors are normally used, but for the purposes of this paper it is sufficient enough to employ diodes). Fig. 15 depicts the network.

In this case, the links are the short circuit transformer inductances included to take into account the non-ideal commutation of the diodes, the load resistance and the short-circuit between each six-pulse rectifier bridge. The diodes are numbered in their traditional firing sequence for each of the six-pulse bridges. Even though diodes are nonlinear elements, they can be modeled as piecewise linear for power system studies. Since their internal details are usually neglected, they can be visualized as a simple switch that is either open or closed [32]. In our studies the diodes are modeled as a high value resistance if open $\left(R_{\text {open }}=1 \mathrm{G} \Omega\right)$ or a low value, if closed $\left(R_{\text {closed }}=0.1 \mathrm{~m} \Omega\right)$. As indicated in Fig. 15, the ac part of the network is the slow sub-
TABLE III

Network Parameters For the NeTWORK SHOWN IN FIG. 15

\begin{tabular}{|c|c|}
\hline Component & Value \\
\hline$R_{g a}=R_{g b}=R_{g c}=R_{g}$ & $2 \Omega$ \\
\hline$L_{g a}=L_{g b}=L_{g c}=L_{g}$ & $30 \mathrm{mH}$ \\
\hline$L_{s a l}=L_{s b l}=L_{s c l}=L_{s a 2}=L_{s b 2}=L_{s c 2}=L_{s}$ & $30 \mathrm{mH}$ \\
\hline$L_{s m}$ & $0.5 \mathrm{H}$ \\
\hline$R_{D C}$ & $5 \Omega$ \\
\hline
\end{tabular}

system and will be simulated with a time step of $500 \mu$ s while the bridge is simulated with the time step of $50 \mu \mathrm{s}$. Another partitioning is performed in order to separate the two six-pulse bridges as also indicated in Fig. 15. The advantage of this segmentation is that the conductance matrices corresponding to all possible valve combinations may be pre-calculated and stored in computer memory [33]. Consequently there is no need to re-triangularize the bridge matrices whenever a switch changes state. The pre-calculated matrix is simply picked up from computer memory based on the results of the previous solution step.

$V_{a}, V_{b}$, and $V_{c}$ form a three-phase balanced voltage source of amplitude $163 \mathrm{kV}$ and frequency $60 \mathrm{~Hz}$. $V_{\mathrm{inv}}$ is included in order to represent the inverter as a constant voltage source of amplitude $484 \mathrm{kV}$ The backward Euler integration rule was adopted for the simulations since it does not introduce numerical oscillations at discontinuities [32], [34]. This is of critical importance in the simulation of power electronics devices. Recently, a method based on a hybrid approach (piecewise transients analysis joined by dc analysis) has been proposed in order to avoid numerical difficulties associated with switching events [35]. This approach is particularly interesting when nonlinear solution methods are employed for the entire network. However, 


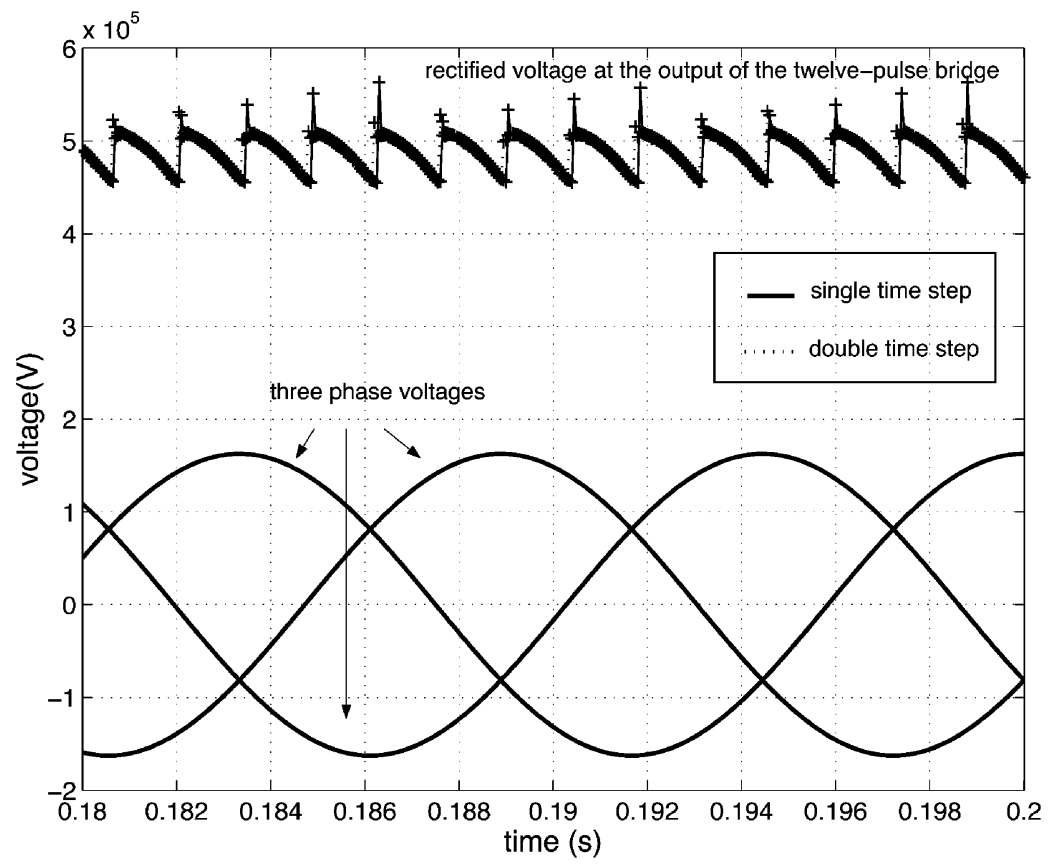

Fig. 16. Three-phase and rectified voltage for the single-time-step ( $\Delta t=50 \mu \mathrm{s})$ and for the double-time-step solutions $(\Delta T=500 \mu \mathrm{s}$ and $\Delta t=50 \mu \mathrm{s})$.

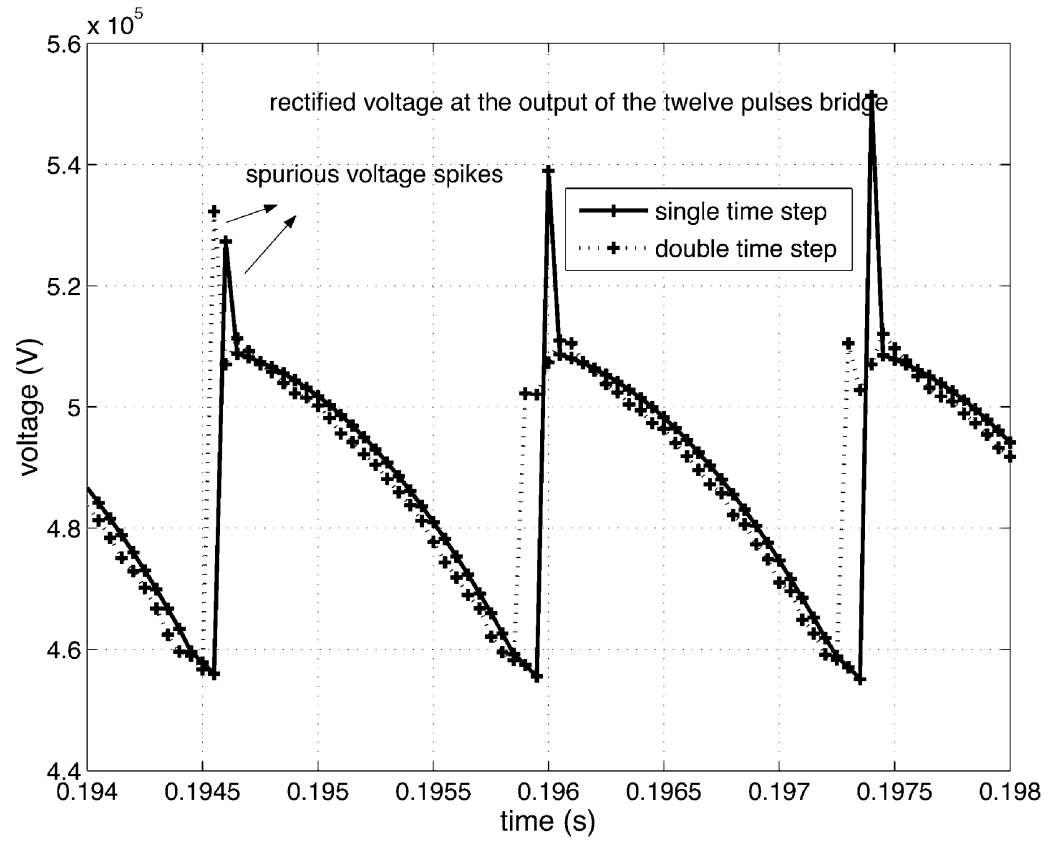

Fig. 17. Rectified voltage for the single-time-step $(\Delta t=50 \mu \mathrm{s})$ and for the double-time-step solutions $(\Delta T=500 \mu \mathrm{s}$ and $\Delta t=50 \mu \mathrm{s})$.

as explained in the Appendix, this is not usually the philosophy followed in power system transient simulators.

Table III shows the values adopted for the network elements.

Fig. 16 presents the three-phase and the rectified voltages obtained when a single time step of $50 \mu \mathrm{s}$ is adopted for the whole network and also when a time step of $50 \mu \mathrm{s}$ is adopted for the rectifier bridge whereas a time step of $500 \mu \mathrm{s}$ is used in the ac part of the network.

Fig. 17 presents the rectified voltage enlarged so as to facilitate the comparison between the traditional approach of a single integration step and the double-time-step methods.
The results obtained with the double-time-step method are in good agreement with the traditional single step approach, except for a very small phase shift and for the spurious voltage spikes that appear in the rectified voltages which are due to the actual commutation happening in-between solution steps. Over the past few years, there have been many contributions in order to eliminate or at least reduce the amplitude of these spurious spikes in simulations. The proposed solutions are usually based on linear interpolation of the current through the diodes so as to detect the zero crossing as precisely as possible [36]-[38]. Its analysis, however, is beyond the scope of this paper. Fig. 18 


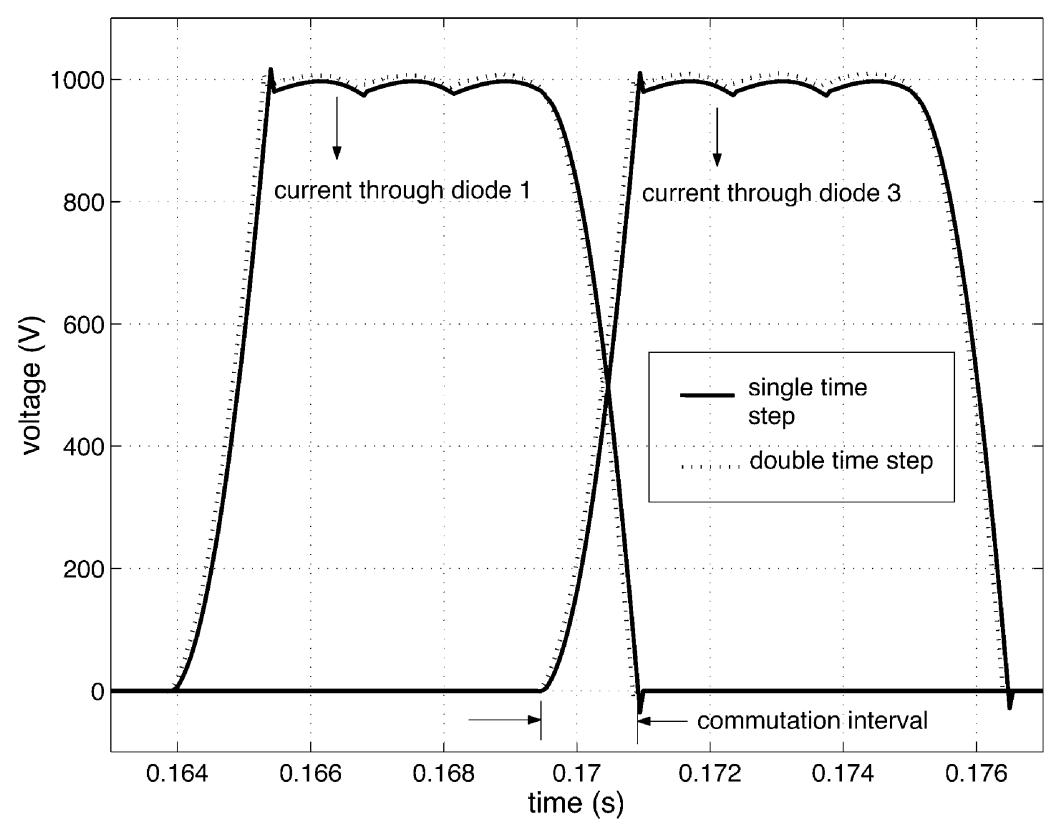

Fig. 18. Current commutation between diodes 1 and 3 for the single-time-step $(\Delta t=50 \mu \mathrm{s})$ and for the double-time-step solutions $(\Delta T=500 \mu \mathrm{s}$ and $\Delta t=$ $50 \mu \mathrm{s})$.

shows the current commutation between diodes 1 and 3 for the single and double-time-step methods.

Once again, the results obtained with both approaches are very similar. Latency exploitation has therefore been validated in terms of accuracy for the rectifier bridge. If the rectifier bridge had been simulated with a time step of $500 \mu \mathrm{s}$, the accuracy of the switching devices would be limited to about 10 electrical degrees, a value completely unacceptable for the simulation of rectifiers and/or inverters.

The whole network contains ten elements for which history sources must be evaluated: three inductances representing the generator reactances, six inductances representing the transformer reactances and one inductance accounting for the smoothing reactance in the dc side of the network. Seven of these history sources must be evaluated at every small integration step $\Delta t$ (the one related to the smoothing reactance and the six related to the transformer reactances, which are also the linking branches between the fast and slow subsystems). Only the three history sources associated with the generator reactances are calculated at every large time step $\Delta T$. In terms of node voltages, the whole network contains twenty three nodes. Twelve of these nodes are located in the slow subnetwork. Meanwhile, in the fast subnetwork, there are six nodes forming the top six-pulse bridge and five nodes forming the bottom six-pulse one (the top bridge has one more node due to the presence of the smoothing reactance).

Table IV shows the number of floating-point operations performed for each situation in order to run the simulation up to $0.2 \mathrm{~s}$. The codes once again have been written in MATLAB version 6 , both for the double- and single-time-step methods for fairness of comparison. Sparsity has been exploited in both cases.

The single-time-step alternative requires approximately $80 \%$ more FLOPS than the double-time-step case. A more significant
TABLE IV

Number OF FLOPS FOR SingLE- AND DOUBLE-TiME-STEP METHOdS. SIMULATION RUN UP TO $0.2 \mathrm{~s}$

\begin{tabular}{|c|c|}
\hline Method & FLOPS \\
\hline Single time step $(\Delta t=50 \mu \mathrm{s})$ & $8,325,314$ \\
\hline Double time step $(\Delta t=50 \mu \mathrm{s}$ and $\Delta T=500 \mu \mathrm{s})$ & $4,681,637$ \\
\hline
\end{tabular}

reduction could be expected, for example, in an HVDC transmission link where both rectifier and inverter are represented as well as the dc transmission line.

\section{CONCLUSION}

This paper presented the concept of latency exploitation within the context of a simultaneous solution without iterations. The implementation is based on the latent behavior of slow subsystems represented with an interpolated Thevenin equivalent. The MATE concept of a common simultaneous solution of the links subsystem which connects individual subsystems that are internally solved with different solution techniques provides the global solution framework. With MATE, a network containing both slow and fast subsystems can be partitioned and distinct integration step sizes can be used for the simulation of each individual subsystem.

In the methodology presented, the contribution of the slow part of the network is taken into account when solving only for the fast part by an interpolation of its Thevenin equivalent voltage source. The variation of the fast subsystem through a complete large time step $\Delta T$ is accounted for by injecting a link current that is the average of all the link currents calculated during the slow subnetwork solution step interval.

Circuit latency has been exploited using robust direct integration methods like backward Euler and trapezoidal instead of the 
more traditional iterative methods, making the approach useful for real-time simulation.

Two electric networks have been simulated using the proposed latency technique. The first case consisted on a network containing lossy transmission lines separating a fast subnetwork from the rest of the system while the second case consisted on a twelve-pulse rectifier bridge where a small time step is employed for the correct simulation of the switching of the diodes, whereas a large time step is used for the ac part of the network. Accuracy was maintained and there was a gain of about $70 \%-80 \%$ in the number of floating-point operations when latency is exploited. Additional improvements are expected for larger size networks.

The MATE simulation method and its associated latency exploitation technique has its main advantage when there are not too many interconnecting links between fast and slow subnetworks. When this is not the case, the processing time for solving the link equations and injecting the link currents into the subnetwork may not be justifiable when compared to a complete network solution using sparsity techniques. For large systems, network partitioning should be performed judiciously. A recent work [39] has focused on the task of efficient network partitioning in order to minimize the number of links derived from the partitioning. This approach should be particularly important in a PC cluster environment where the network partitioning could be optimized for an efficient task assignment to each processor.

For future work, the authors are considering latency exploitation in networks containing truly nonlinear elements. If the nonlinear elements can be grouped into subsystems through the MATE technique, then only these nonlinear subnetworks would need to be solved iteratively, for example using the Newton-Raphson method. In the Appendix, the necessary steps for the inclusion of nonlinearities for latency exploitation under the MATE framework are described. The authors also intend to exploit latency in other mixed-solution environments, such as when subsystems are solved with different integration techniques [40]. For example, if all important electrical information in one or more subnetworks is steady-state and it can be adequately solved using a quasi-steady-state phasor solution (as normally done in machine stability studies), while a traditional transient solution is required for a particular subnetwork, then considerable savings in simulation time can be achieved.

\section{APPENDIX}

The purpose of this section is to discuss the possible inclusion of nonlinear elements for latency exploitation within the framework provided by the MATE technique. Even though the examples presented in this paper are limited to linear or piece-wise linear systems, as in the ideal modeling of diodes, true nonlinearities can be treated in a relatively straight forward way for latency exploitation under the proposed method. In power system transients studies the network usually contains only a few nonlinear elements generally with the purpose of modeling metal oxide surge arresters and saturation in transformers and reactors. It is therefore more sensible to modify the well-proven linear methods to accommodate nonlinear elements than to use less efficient nonlinear solution methods for the entire network. This is

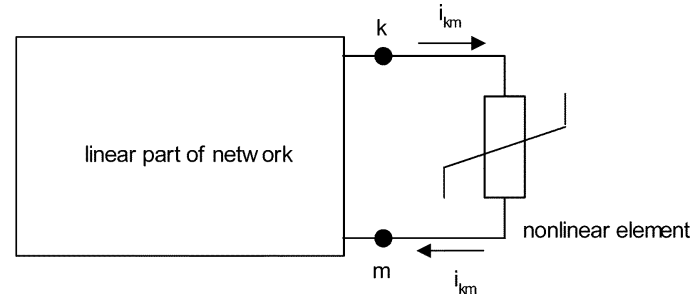

Fig. 19. One nonlinear element connected to linear network.

the philosophy that has been followed in EMTP-type simulators [26]. One of the schemes that has been used with great success is the compensation method [41], which is well adapted to the MATE concept.

In compensation-based methods, the nonlinear elements are essentially simulated as current injections which are superimposed on the linear network after a solution without the nonlinear elements has first been found. There may be some rare situations where a network solution without the nonlinearity is impossible (when no open circuit solution exists). Such cases become solvable if the nonlinear branch is split into two parallel branches, one with a linear characteristic to permit the open circuit solution, and the other with a modified nonlinear branch.

If compensation-based methods are used for the inclusion of nonlinearities then it is possible to exploit latency by following the method presented in the paper either if nonlinear elements are part of the slow or fast subnetworks.

Initially, for illustration, only one nonlinear element is considered in a subnetwork. The inclusion of more nonlinearities is, however, quite straight forward and will be discussed subsequently. Fig. 19 shows one nonlinear element connected to a linear network at nodes $k$ and $m$.

This nonlinear element may form one particular subnetwork by itself or it may be part of a larger subnetwork containing linear elements as well. The compensation theorem states that this nonlinear branch can be excluded from the network, and be simulated as a current source $i$ instead. This source leaves node $k$ and enters node $m$ if the nonlinear element is treated as a load. From a general point of view, the nonlinear element may be considered as a sub-subnetwork within an original subnetwork. The current $i_{k m}$ must fulfill two equations, namely the network equations of the linear part, which is the instantaneous Thevenin equivalent circuit between nodes $k$ and $m$

$$
v_{k m}(t)=v_{k m-0}(t)-R_{\mathrm{th}, k m} i_{k m}(t)
$$

where subscript " 0 " indicates solution without the nonlinear branch, and the relationship of the nonlinear branch itself

$$
v_{k m}(t)=f\left(i_{k m}(t), \frac{d i_{k m}(t)}{d t}, t, \ldots\right) .
$$

The solution without the nonlinear branch is in fact one of the steps performed in the MATE solution, equivalent to the solution of the subnetworks considering them completely decoupled (open circuit voltage $v_{k m-0}(t)=v_{k-0}(t)-v_{m-0}(t)$ ), and the Thevenin equivalent resistance between nodes $k$ and $m R_{\mathrm{th}, k m}$ also results directly from the MATE formulation as shown in 
Section II. Equations (16) and (17) are then solved simultaneously for $i_{k m}$ using, for example, Newton-Raphson's method. The final solution is then found by superimposing the open-circuit solution to the link current $i_{k m}$ also in the way previously discussed in the MATE formulation

$$
v_{A}(t)=v_{A-0}(t)-R_{\mathrm{th}} i_{k m}(t)
$$

where

$\boldsymbol{v}_{A} \quad$ vector of node voltages;

$v_{A-0}$ vector of node voltages without the nonlinear branch;

$\boldsymbol{R}_{\mathrm{th}} \quad$ vector of Thevenin equivalent resistances.

If there are two or more nonlinear elements in a subnetwork, then $M$ Thevenin equivalent resistances for each pair of nodes $k_{i}, m_{i}$ connected to the nonlinear elements must first be found (where $M$ is the number of nonlinear elements, and $i$ varies from 1 to $M$ ). The solution without the nonlinear branches is calculated in the same way as before but now the simultaneous solution of $M$ nonlinear equations is required

$$
v_{k m}(t)=v_{k m-0}(t)-R_{\mathrm{th}, k m} i_{k m}(t)
$$

where $\boldsymbol{v}_{k m}, \boldsymbol{v}_{k m-0}$, and $\boldsymbol{i}_{k m}$ are $m \times 1$ vectors and $\boldsymbol{R}_{\mathrm{th}, k m}$ is an $m \times m$ matrix.

The final solution is then found by superimposing the link currents $\boldsymbol{i}_{k m}$

$$
\begin{array}{r}
v_{A}(t)=v_{A-0}(t)-R_{\mathrm{th}}^{(1)} i_{k m}^{(1)}(t)-R_{\mathrm{th}}^{(2)} i_{k m}^{(2)}(t) \\
-\ldots R_{\mathrm{th}}^{(m)} i_{k m}^{(m)}(t)
\end{array}
$$

where $R_{\mathrm{th}}^{(1)}, R_{\mathrm{th}}^{(2)}, \ldots, R_{\mathrm{th}}^{(M)}$ are $N \times 1$ vectors, where $N$ is the number of nodes in the subnetwork

Once again, the solution can be obtained in a straight forward way by following the MATE formulation and using the Newton-Raphson method for the simultaneous solution of the nonlinear equations.

\section{REFERENCES}

[1] L. W. Nagel and D. O. Pederson, "Simulation program with integrated circuit emphasis," in Proc. 16th Midwest Symp. Circuits Theory, Waterloo, Canada, Apr. 1973.

[2] H. W. Dommel, "Digital computer solution of electromagnetic transients in single and multiphase networks," IEEE Trans. Power App. Syst., vol. 88, no. 4, pp. 388-399, Apr. 1969.

[3] R. A. Saleh and A. R. Newton, "The exploitation of latency and multirate behavior using nonlinear relaxation for circuit simulation," IEEE Trans. Comput.-Aided Design, vol. 8, no. 12, pp. 1286-1298, Dec. 1989.

[4] E. Lelarasmee, A. E. Ruehli, and A. L. Sangiovanni-Vincentelli, "The waveform relaxation method for time-domain analysis of large integrated circuits," IEEE Trans. Comput.-Aided Design, vol. CAD-1, pp. 131-145, Jul. 1982.

[5] A. I. Zecevic and N. Gacic, "A partitioning algorithm for the parallel solution of differential-algebraic equations by waveform relaxation," IEEE Trans. Circuits Syst. I, Fundam. Theory Appl., vol. 46, no. 4, pp. 421-434, Apr. 1999.

[6] M. Ilic-Spong, M. L. Crow, and M. A. Pai, "Transient stability simulation by waveform relaxation methods," IEEE Trans. Power Syst., vol. PS-2, no. 4, pp. 943-952, Nov. 1987.

[7] M. L. Crow and M. Ilic, "The parallel implementation of the waveform relaxation method for transient stability simulations," IEEE Trans. Power Syst., vol. 5, no. 3, pp. 922-932, Aug. 1990.

[8] P. G. McLaren, R. Kuffel, R. Wierckx, J. Giesbrecht, and L. Arendt, "A real-time digital simulator for testing relays," IEEE Trans. Power Delivery, vol. 7, no. 1, pp. 207-213, Jan. 1992.
[9] M. Kezunovic, M. Aganagic, V. Skendzic, J. Domaszewicz, J. K. Bladow, D. M. Hamai, and S. M. McKenna, "Transients computation for relay testing in real-time," IEEE Trans. Power Delivery, vol. 9, no. 3, pp. 1298-1307, Jul. 1994.

[10] J. R. Martí and L. R. Linares, "Real-time EMTP-based transients simulation," IEEE Trans. Power Systems, vol. 9, no. 3, pp. 1309-1317, Aug. 1994.

[11] J. A. Hollman and J. R. Martí, "Real-time network simulation with PC-clusters," IEEE Trans. Power Syst., vol. 18, no. 2, pp. 563-569, May 2003

[12] P. F. Cox, R. G. Burch, D. E. Hocevar, P. Yang, and B. D. Epler, "Direct circuit simulation algorithms for parallel processing," IEEE Trans. Comput.-Aided Design, vol. 10, no. 6, pp. 714-725, Jun. 1991.

[13] K. A. Sakallah and S. W. Director, "SAMSON2: An event driven VLSI circuit simulator," IEEE Trans. Comput.-Aided Design, vol. CAD-4, pp. 668-685, Oct. 1985.

[14] P. F. Cox, R. G. Burch, P. Yang, and D. E. Hocevar, "New implicit integration method for efficient latency exploitation in circuit simulation," IEEE Trans. Comput.-Aided Design, vol. 8, no. 10, pp. 1051-1064, Oct. 1989.

[15] M. L. Crow and J. G. Chen, "The multirate method for simulation of power system dynamics," IEEE Trans. Power Syst., vol. 9, no. 3, pp. 1684-1690, Aug. 1994.

[16] M. L. Crow and J. G. Chen, "The multirate simulation of FACTS devices in power system dynamics," IEEE Trans. Power Syst., vol. 11, no. 1, pp. 376-382, Feb. 1996.

[17] A. Semlyen and F. de León, "Computation of electromagnetic transients using dual or multiple time steps," IEEE Trans. Power Syst., vol. 8, no. 3, pp. 1274-1281, Aug. 1993.

[18] A. Kurita, H. Okubo, K. Oki, S. Agematsu, D. B. Klapper, N. W. Miller, W. W. Price, J. J. Sanchez-Gasca, K. A. Wirgau, and T. D. Younkins, "Multiple time-scale power system dynamic simulation," IEEE Trans. Power Syst., vol. 8, no. 1, pp. 216-223, Feb. 1993.

[19] T. Kato and K. Ikeuchi, "Variable order and variable step-size integration method for transient analysis programs," IEEE Trans. Power Syst., vol. 6, no. 1, pp. 206-213, Feb. 1991.

[20] J. R. Martí, L. R. Linares, J. Calviño, H. W. Dommel, and J. Lin, "OVNI: An object approach to real-time power system simulators," in Proc. 1998 Int. Conf. Power Syst. Technol. (Powercon), Beijing, China, pp. 977-981.

[21] L. R. Linares, "OVNI, object virtual network integrator: A new fast algorithm for the simulation of very large electric networks in real time," Ph.D. dissertation, Dept. Elect. Comput. Eng., Univ. British Columbia, Vancouver, BC, Canada, 2000.

[22] J. R. Martí, L. R. Linares, J. A. Hollman, and F. A. Moreira, "OVNI: Integrated software/hardware solution for real-time simulation of large power systems," in Proc. 2002 Power Syst. Comput. Conf. (PSCC'02), Seville, Spain, 2002 [Online]. Available: http://www.pscc02.org, Session 33, Paper 4.

[23] G. Kron, "Tensorial analysis of integrated transmission systems, part III-The primitive division," AIEE Trans., vol. 71, pt. 3, pp. 814-821, 1952.

[24] G. Kron, Diakoptics-Piecewise Solution of Large-Scale Systems. London, U.K.: MacDonald, 1963.

[25] C. W. Ho, A. E. Ruehli, and P. A. Brennan, "The modified nodal approach to network analysis," IEEE Trans. Circuits Syst., vol. CAS-22, pp. 504-509, Jun. 1975.

[26] H. W. Dommel, EMTP Theory Book, 2nd ed. : Microtran Power System Analysis Corporation, 1996, pp. 12-3-12-9.

[27] F. A. Moreira, "Latency techniques in power systems transients simulation," Ph.D. dissertation, Dept. Elect. Comp. Eng., Univ. British Columbia, Vancouver, BC, Canada, 2002.

[28] F. A. Moreira and J. R. Martí, "Latency techniques for time-domain power system transients simulation," IEEE Trans. Power Syst., vol. 20, no. 1, pp. 246-253, Feb. 2005.

[29] F. A. Moreira and J. R. Martí, "Latency suitability for the time-domain simulation of electromagnetic transients through network eigenanalysis," in Proc. 2003 Int. Conf. Power System Transients (IPST), New Orleans, LA [Online]. Available: http://www.ipst.org/IPST03Papers.htm, Session 3a, Paper 4.

[30] S. D. Pekarek, O. Wasynczuk, E. A. Walters, J. V. Jatskevich, C. E. Lucas, N. Wu, and P. T. Lamm, "An efficient multirate simulation technique for power-electronic-based systems," IEEE Trans. Power Syst., vol. 19, no. 1, pp. 399-409, Feb. 2004.

[31] A. M. Gole and V. K. Sood, "A static compensator model for use with electromagnetic transients simulation programs," IEEE Trans. Power Delivery, vol. 5, no. 3, pp. 1398-1407, Jul. 1990. 
[32] J. Vlach and A. Opal, "Modern CAD methods for analysis of switched networks," IEEE Trans. Circuits Syst. I, Fundam. Theory Appl., vol. 44, no. 8, pp. 759-762, Aug. 1997.

[33] S. Acevedo, L. R. Linares, J. R. Martí, and Y. Fujimoto, "Efficient HVDC converter model for real-time transients simulation," IEEE Trans. Power Syst., vol. 14, no. 1, pp. 166-171, Feb. 1999.

[34] J. R. Martí and J. Lin, "Suppression of numerical oscillations in the EMTP,” IEEE Trans. Power Syst., vol. 4, no. 2, pp. 739-747, May 1989.

[35] E. Chiarantoni, G. Fornarelli, S. Vergura, and T. Politi, "Applying fixed point homotopy to nonlinear DAEs deriving from switching circuits," Future Generation Comput. Syst., vol. 19, no. 3, pp. 431-441, Apr. 2003.

[36] P. Kuffel, K. Kent, and G. Irwin, "The implementation and effectiveness of linear interpolation within digital simulation," Int. J. Elect. Power Energy Syst., vol. 19, no. 4, pp. 221-227, May 1997.

[37] K. Strunz, L. Linares, J. R. Martí, O. Huet, and X. Lombard, "Efficient and accurate representation of asynchronous network structure changing phenomena in digital real-time simulators," IEEE Trans. Power Syst., vol. 15, no. 2, pp. 586-592, May 2000.

[38] K. Strunz, "Flexible numerical integration for efficient representation of switching in real-time electromagnetic transients simulation," IEEE Trans. Power Delivery, vol. 19, no. 3, pp. 1276-1283, Jul. 2004

[39] P. Zhang, J. R. Martí, and H. W. Dommel, "Network partitioning for real-time power system simulation," in Proc. 2005 Int. Conf. Power System Transients (IPST'05), Montreal, QC, Canada, 2005 [Online]. Available: http://www.ipst.org/IPST05Papers.htm, Session 11, Paper 177.

[40] M. Sultan, J. Reeve, and R. Adapa, "Combined transient and dynamic analysis of HVDC and FACTS systems," IEEE Trans. Power Delivery, vol. 13, no. 4, pp. 1271-1277, Oct. 1998.

[41] H. W. Dommel, "Nonlinear and time-varying elements in digital simulation of electromagnetic transients," IEEE Trans. Power App. Syst. vol. 90, no. 6, pp. 2561-2567, Nov. 1971.

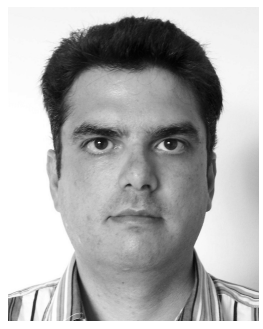

Fernando A. Moreira (S'98-M'03) was born in São Paulo, Brazil, in 1970. He received the B.Sc. and M.Sc. degrees both in electrical engineering from the University of São Paulo, São Paulo, Brazil in 1994 and 1997, respectively, and the Ph.D. degree also in electrical engineering from the University of British Columbia, Vancouver, BC, Canada in 2002.

From 2003 to 2005, he was a Post-Doctoral Fellow at the University of São Paulo. Currently he is an Assistant Professor at the Federal University of Bahia, Salvador, Brazil. His research interests are solution methods and modeling of transmission lines and power electronic devices for electromagnetic transients programs.

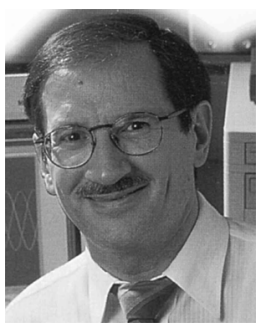

José R. Martí (M'79-F'02) received the B.S. degree in electrical engineering from Central University of Venezuela, Caracas, Venezuela, the M.E.E.P.E. degree from Rensselaer Polytechnic Institute, Troy, NY, and the Ph.D. degree from the University of British Columbia, Vancouver, BC, Canada, in 1971, 1974, and 1981, respectively.

He has made contributions in modeling of transmission lines, transformers, and in new solution techniques for off-line and real-time applications. He is a Professor at the University of British Columbia.

Dr. Martí is a Registered Professional Engineer in the Province of British Columbia.

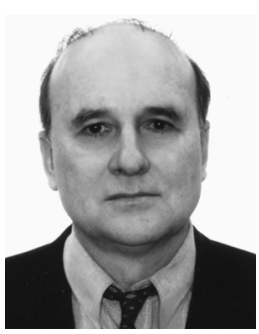

Luiz C. Zanetta, Jr. (SM'90) was born in 1951 in Brazil. He received the B.Sc., M.Sc, and Ph.D. degrees from Escola Politécnica da Universidade de São Paulo (EPUSP), São Paulo Brazil, in 1974, 1984 , and 1989 , respectively.

Currently, he is a Professor at EPUSP carrying out research in the fields of electrical system dynamics and electromagnetic transients. He joined THEMAG Engenharia Ltd., São Paulo, Brazil, in 1975, and developed power system studies for most of the Brazilian utilities until 1989.

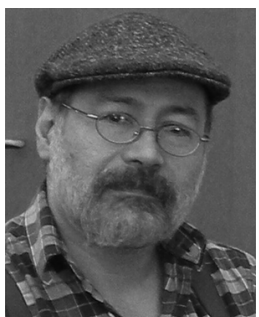

Luis R. Linares received the B.S. degree in electrical engineering from Central University of Venezuela, Caracas, Venezuela, and the M.A.Sc and Ph.D. degrees from the University of British Columbia, Vancouver, BC, Canada, in 1981, 1993, and 2000, respectively.

He has made contributions in real-time simulation of fast transients of power electric networks. He has been a Faculty Member with the University of British Columbia since January 2001. 